\title{
Y123 相超导体有机保护膜研究
}

\author{
张春波曹必松 何艾生 ${ }^{\circledR}$ 朱美红赵永刚
}

(清华大学物理系, 北京 100084, (1) 北方工业大学, 北京 100041)

\section{关链词高温超导体 有机保护膜 $\mathrm{pH}$ 值}

高温超导材料在实用中要求其超导性能稳定. 但是, $\mathrm{Y}$ 系超导材料易受环境中的水汽、 $\mathrm{CO}_{2}$ 侵蚀而分解, 生成的最终产物含有 $\mathrm{Ba}(\mathrm{OH})_{2}, \mathrm{Y}(\mathrm{OH})_{3}$ 以及 $\mathrm{BaCO}_{3}$ 等碱性物质 ${ }^{[1 \sim 0 ;}$; 一些超导器 件 (如超导微波器件) 需防止器件与环境的各种接触造成表面性能恶化. 因此, 有必要对超导 器件进行保护, 与各种不良环境隔离. 国外有过一些使用无机保护材料的报道 ${ }^{[7 \sim 10]}$, 主要采用 真空热蒸发沉积和酸钝化方法, 可将超导材料在水中保护约数分钟到 $1 \mathrm{~h}$; 文献 [1] 用有机保 护材料浸渍超导材料, 可获得在水中保护数小时的结果. 本文用 $\mathrm{pH}$ 酸度计进行监测, 选用有 机高分子化合物作保护材料, 可在水中保护 $40 \mathrm{~h}$ 以上.

\section{1 实验方法}

用普通固相反应方法高温烧结得到 $\mathrm{Y}_{1} \mathrm{Ba}_{2} \mathrm{Cu}_{3} \mathrm{O}_{7-x}$ 块材, 其 $\mathrm{X}$ 射线衍射谱见图 1 谱线 1 .

选用有机玻璃和性能优良的 45 号有机膜作保护材料, 通过离心镀膜法在超导块材表面形 成均匀致密的表面保护膜. 实验中超导块材仅在一面铰保护膜, 其余数面密封, 镀保护膜的 一面先打磨平滑. 保护膜厚度用千分表测量. 将涂有保护膜的 Y123 相超导块材浸泡于去离 子水中, 用 $\mathrm{pH}$ 酸度计检测水中 $\mathrm{pH}$ 值变化, 以了解超导体与水反应情况, 从而不间断地监测 保护效果. 并测量浸水前后超导体 $T_{\mathrm{c}}$ 和 $J_{c}$ 的变化. 最后对超导天线加保护膜进行天线微波特 性测量, 以检验所用保护膜对器件性能影响及其对器件性能的稳定作用.

\section{2 实验结果及分析}

首先需要了解镀膜溶液对超导材料有无损害, 为此将超导粉与镀膜溶液混合, 待溶剂蒸发 干后测出 $X$ 射线衍射谱 (XRD). 因为高分子 XRD 相对超导体 XRD 而言只是一些较小起 伏, 且没有尖锐峰, 把它们与超导体 XRD 叠加只对超导体 XRD 峰强略有影响, 对峰位无影 响, 因此可将测得的 XRD 与超导体的 XRD 比较, 通过了解有无异相峰出现以及峰强是否有 较大变化, 来判断铰膜溶液对超导体的影响. 图 1 的 XRD 表明有机玻璃溶液及 45 号有机膜 溶液对 Y123 相超导体基本无影响. 图 2 为无保护的 Y123 相超导块材浸水后水的 $\mathrm{pH}$ 值随 时间 $t$ 变化的 $\mathrm{pH}-t$ 曲线, 去离子水量为 $40 \mathrm{~mL}$, 超导块裸露面积约 $0.5 \mathrm{~cm}^{2}$, 本文中以下提到 的浸水实验所用条件与此相同, 不同之处仅在于裸露面是否涂有保护膜. 曲线中一开始 $\mathrm{pH}$ 值 小于 7 是因为所用去离子水中溶人了 $\mathrm{CO}_{2}$ 的缘故 (下同), 对实验结果的相对变化无影响. 曲 线 2 用的是放置了两个月的超导材料, $5 \sim 6 \mathrm{~min}$ 内 $\mathrm{pH}$ 值迅速升到 10 左右, 然后缓慢地在 $24 \mathrm{~h}$ 后升到 11.7(图上没有画出), 可见该超导材料浸水前已经受到了空气侵蚀生成了一些碱性物

1994-08-15 收稿, 1995-03-06 收修改稿 
质. 曲线 1 用的是刚烧出的超导材料, 没受到长时间空气侵蚀, 因此 $\mathrm{pH}$ 值上升略缓, 在 $10 \mathrm{~min}$ 内只升到 $8,1.5 \mathrm{~h}$ 左右升到 9.

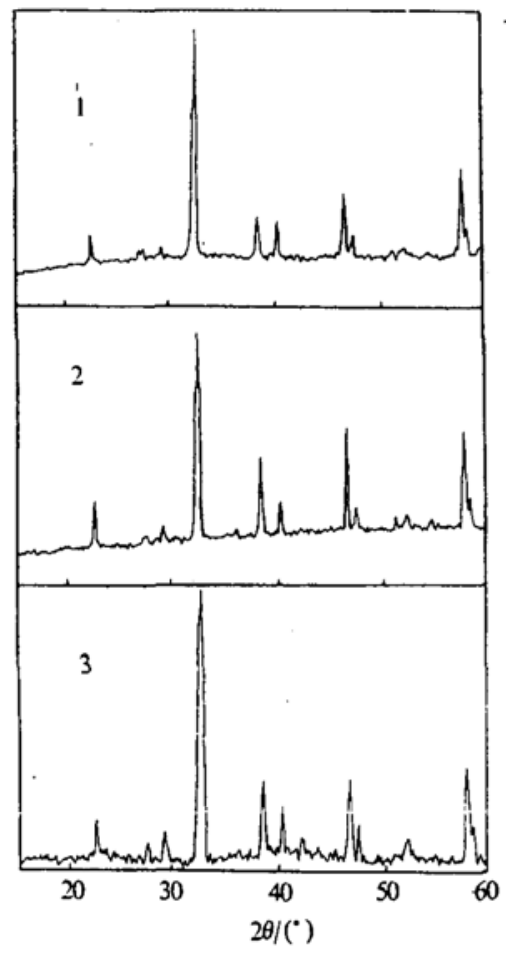

图 1 Y123 相超导体

1 为超导体的 XRD, 2 为超导材料与有机玻瑞 溶液混合物的 XRD, 3 为超导材料与 45 号有 机膜溶液混合物的 XRD

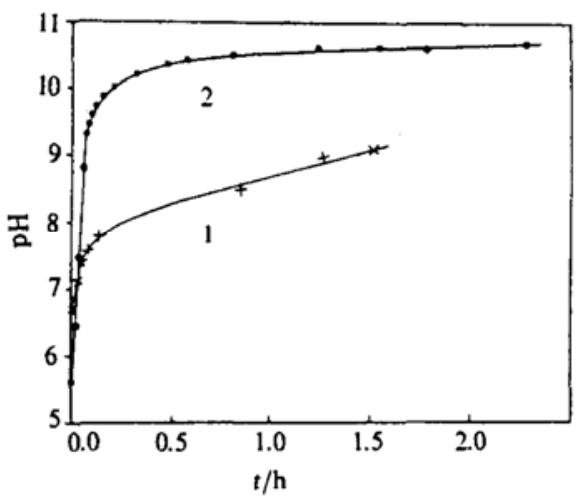

图 2 无保护的 Y123 相超导块材的浸水 $\mathrm{pH}-t$ 曲线 曲线 1 用的是刚烧出的超导材料,曲线 2 用的是放置了 2 个 月的超导材料

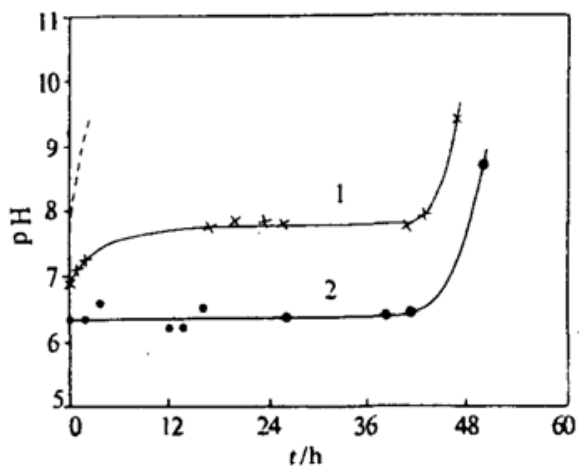

图 3 涂有保护膜的 Y123 相超导材料的浸水 $\mathrm{pH}-t$ 曲线 虚线为图 2 曲线 1 的结果. 曲线 1 用厚约 $17 \mu \mathrm{m}$ 的 45 号有机 膜保护, 曲线 2 用厚约 $15 \mu \mathrm{m}$ 的有机玻瑞膜保护

可由溶解度及水解常数估算得 $\mathrm{BaCO}_{3}, \mathrm{Y}(\mathrm{OH})_{3}$ 及 $\mathrm{Ba}(\mathrm{OH})_{2}$ 达到溶解平衡后其饱和溶液的 $\mathrm{pH}$ 值分别约为 $10.1,12$ 及 13.7. 由于去离子水中溶有 $\mathrm{CO}_{2}$, 会 使实际的 $\mathrm{BaCO}_{3}$ 的饱和溶液 $\mathrm{pH}$ 值小于 10.1 , 但大致在 10 左右. 可见当生成的碱性物质全溶解在水中但还未形成饱和溶 液时, 溶液的 $\mathrm{pH}$ 值约小于 10 . 当 $\mathrm{pH}=10$ 时 $\mathrm{OH}^{-}$的浓度为 $10^{-4} \mathrm{~mol} / \mathrm{L}$, 水中阳离子浓度约等 于 $\mathrm{OH}^{-}$的浓度. 阳离子的各原子的原子量和约在 100 量级. 此时在 $100 \mathrm{~g}$ 水 $(0.1 \mathrm{~L})$ 中溶解的 碱性物质约有 $10^{-4} \mathrm{~mol} / \mathrm{L} \times 0.1 \mathrm{~L} \times 100 \mathrm{~g} / \mathrm{mol}=1 \mathrm{mg}$, 则相应的反应掉的超导体的量也在毫克量 级. 同理可知, $\mathrm{pH}$ 值在 $7 \sim 8$ 间时在 $100 \mathrm{~g}$ 水中反应掉的超导体的量仅约 $10^{-5} \sim 10^{-6} \mathrm{~g}$ 量 级. 可见, 使用 $\mathrm{pH}$ 酸度计可探测到微量的超导体与水反应情况, 是一种精度较高的和较方便 的检测方法.

上面的估算是在假设超导体与水反应迅速得到最终产物 $\mathrm{BaCO}_{3}$ 等时进行的, 即认为反应 很快, 中间产物 (酸碱性不明的产物)不致过多积累. 如果考虑中间产物, 则应将估算值当作 
实际值的下限. 文献 [5] 等得出 $\mathrm{Y} 123$ 相超导体与水、 $\mathrm{CO}_{2}$ 反应较快地明显生成 $\mathrm{BaCO}_{3}$, 保证 了上述假设在一定程度上成立.

图 3 为有保护膜的超导块材的浸水 $\mathrm{pH}-t$ 曲线, 可见 $\mathrm{pH}$ 值均基本稳定了约 $40 \mathrm{~h}$, 与无保 护时的结果相比较可知, 涂了保护膜的样品的 $\mathrm{pH}$ 值的变化大大延缓了. 其中曲线 1 一开始在 7 左右升了约 0.8 , 这估计是未能彻底杜绝保护膜上的微细孔所致, 按估算此时与水反应掉的 超导体的量仅在 $10^{-5} \sim 10^{-6} \mathrm{~g}$ 量级, 对超导性能不构成明显影响. 因此据图认为涂有保护膜 的 Y123 相块材可在水中被保护约 $40 \mathrm{~h}$, 超导体未受到明显侵蚀.

为了得到保护膜在空气中的实际保护效果, 需要将水中的有效保护时间折算成空气中的 有效保护时间, 折算方法是比较单位时间在水中和在饱和水汽中水分子对材料单位面积的碰 撞次数, 在水和水汽中, 取十分粗略的近似, 忽略分子相互作用势能, 则碰撞次数均约为 $N=$ $\frac{1}{4} n \bar{v}$, 其中 $n$ 为单位体积分子数密度, $\bar{v}$ 为分子平均速度. 水和饱和水汽两者保持动态平 衡, 两者的 $\bar{v}$ 数值相当 (饱和水汽的略大), 则有

$$
\frac{N_{\text {水 }}}{N_{\text {学 }}} \approx \frac{n_{\text {水 }}}{n_{\text {党 }}}=\frac{\rho_{\text {水 }}}{\rho_{\text {党 }}} \approx 1000 \text {, }
$$

即水中保护 $1 \mathrm{~s}$ 约相当于饱和水汽中保护 $1000 \mathrm{~s}$, 由此粗略估算得, 在水中保护 $40 \mathrm{~h}$ 约相当于 在空气中保护 $4 \sim 5 \mathrm{a}$.

Y123 相超导块材在由液氮温区回复室温过程中由于凝结水的影响, 超导性能会下降, 图 4-1是针对此情况进行实验得到的结果, 此实验同时还有检验保护膜在低温下工作情况 的目的; 图 4-2 是在潮湿空气中进行保护实验得到的结果. 可见经过严酷的条件后保护膜都 在水中将块材保护了 $24 \mathrm{~h}$ 以上. 将图 4-1 的超导块从水中取出测得 $T_{\mathrm{c}}$ 为 $92 \mathrm{~K}, J_{\mathrm{c}}$ 为 $110 \mathrm{~A} / \mathrm{cm}^{2}$, 同批超导块在刚出炉时测得 $T_{\mathrm{c}}$ 为 $92 \mathrm{~K}, J_{\mathrm{c}}$ 为 $100 \mathrm{~A} / \mathrm{cm}^{2}$ 左右, 可见涂了保护膜的超导材料经液 氮和水浸泡后其超导性能基本未变. 对 Y123 相超导薄膜也进行了涂保护膜的初步实验, 图 5 的结果显示保护膜延缓了超导薄膜与水的反应.

最后, 在 Y 123 相超导天线上涂了厚约 $17 \mu \mathrm{m}$ 的 45 号有机膜后进行微波特性测量, 结果显示 保护层对天线性能影响小 (対增益的影响在 $0.1 \mathrm{~dB}$ 左右); 未加保护层的超导天线经过低温循

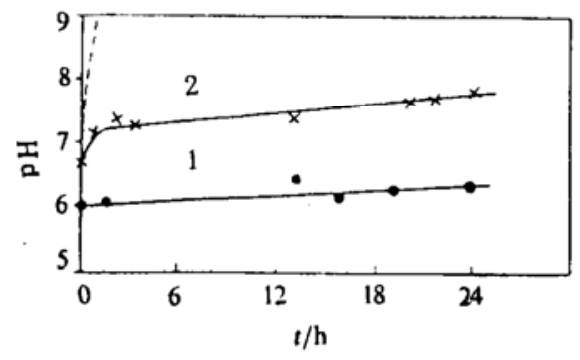

图 445 号有机膜的 Y123 相超导体的 保护实验膜厚约为 $17 \mu \mathrm{m}$

1 为超导材料直接浸人液氛后的浸水 $\mathrm{pH}-t$ 曲线, 2 为超导材料放人 $84 \% \sim 91 \%$ 湿度水汽中 $7 \mathrm{~d}$ 后 的漫水 $\mathrm{pH}-t$ 曲线. 虚线为图 2 曲线 1 的结果

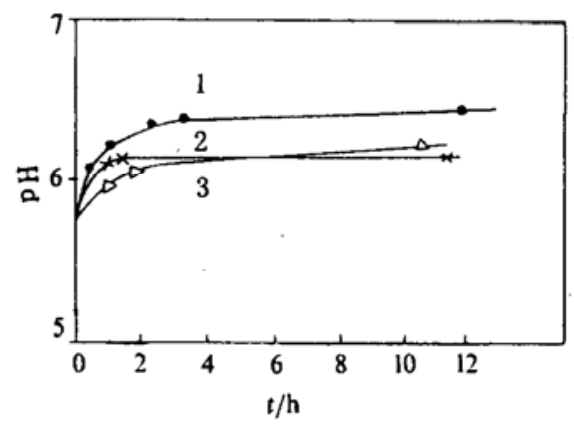

图 $5 \mathrm{Y} 123$ 相超导薄膜的浸水 $\mathrm{pH}-t$ 实验结果 1 为无保护情况, 2 为涂有约 $3 \mu \mathrm{m}$ 厚 45 号有机膜情况, 3 为涂有约 $6 \mu \mathrm{m}$ 厚 45 号有机膜情况 
环后增益会损失 $2 \sim 3 \mathrm{~dB}$, 而多次重复测量的结果表明有保护层的天线增益是稳定的.

上述实验结果初步显示了所用的两种有机保护膜有较好的保护效果, 但仍需继续改善保 护材料和实验方法, 以使实验结果有较好的稳定性.

实验结果表明, 涂有厚约 $17 \mu \mathrm{m}$ 的 45 号有机膜和厚约 $15 \mu \mathrm{m}$ 的有机玻璃膜的 Y123 相超 导块材能在水中被保护 $40 \mathrm{~h}$ 以上, 超导性能不发生明显变化. 电学测量亦表明被保护的 Y123 相超导材料在水中浸泡 $24 \mathrm{~h}$ 后其 $T_{\mathrm{c}}$ 和 $J_{\mathrm{c}}$ 亦无明显变化. 实验同时表明 45 号有机膜有较好 的实际保护效果和低温工作特性, 超导天线保护实验表明该有机膜对器件性能影响小且使器 件性能稳定.

\section{参考文献}

1 Barns R L, Laudise R A. Stability of superconducting $\mathrm{YBa}_{2} \mathrm{Cu}_{3} \mathrm{O}_{7}$ in the presence of water. Appl Phys Lett, 1987, 51(17): 1373

2 郑思定, 曹 媇, 缪柏林等. 环境气氛对 Y-Ba-Cu-O 超导体的影响. 低温物理学报, 1989, 11(3): 218

3 杨宏川,刘文庆, 吴美英等. $\mathrm{YBaCuO}$ 超导体的不稳定性及超导性能敃复. 低温物理学报, 1990, 12(2): 140

4 金继荣,金 新, 陈武鸣等. 水份对 $\mathrm{YBaCuO}$ 超导电性的影响. 低温物理学报, 1990, 12(1): 72

5 周和平, 王 英. $\mathrm{YBa}_{2} \mathrm{Cu}_{3} \mathrm{O}_{7-\mathrm{x}}$ 超导体材料的水解性质. 见: 全国超导学术会议论文集 (1), 宝鸡: 宝鸡稀有金属加工研 究所出版, 1988. 82

6 Wada O, Odaka T, Wakata $\mathrm{M}$ et al. Transmission electron microscopy study of the environmental degradation in $\mathrm{Ba}_{2} \mathrm{YCu}_{3} \mathrm{O}_{7-r}$ J Appl Phys, 1990, 68(10): 5283

7 Ichikawa $\mathrm{Y}$, Adachi $\mathrm{H}$. Effect of overcoating with dielectric films on the superconductive properties of the high- $T_{\mathrm{c}}$ Y-Ba-Cu-O films. Jpn J Appl Phys, 1988, 27(3): 381

8 Hill D M, Meyer H M, Weaver $\mathrm{J} \mathrm{H}$. Passivation of high $T_{\mathrm{c}}$ superconductor surfaces with $\mathrm{CaF}_{2}$ and $\mathrm{Bi}, \mathrm{Al}$, and $\mathrm{Si}$ oxides. Appl Phys Lett, 1988, 53(17): 1657

9 Berkley D D, Broussard P R, Ervin A M. Surface characterization of $\mathrm{YBa}_{2} \mathrm{Cu}_{3} \mathrm{O}_{7-x}$ thin silm supporting metallic and insulating overlayers. IEEE Trans on Mag, 1991, 27(2): 966

10 Vasquez R P, Hunt B D, Foote M C. Wet Chemical Passivation of $\mathrm{YBa}_{2} \mathrm{Cu}_{3} \mathrm{O}_{7-\mathrm{x}}$. J Electrochem Soc, 1990, 137(7): 2344 